\title{
The Solvability and Optimal Controls for Some Fractional Impulsive Equation
}

\author{
Xianghu Liu, ${ }^{1}$ Zhenhai Liu, ${ }^{2}$ and Jiangfeng $\operatorname{Han}^{3}$ \\ ${ }^{1}$ School of Mathematics and Statistics, Central South University, Changsha, Hunan 410075, China \\ ${ }^{2}$ College of Sciences, Guangxi University for Nationalities Nanning, Guangxi 530006, China \\ ${ }^{3}$ Faculty of Mathematics and Computer Science, Jagiellonian University, ul. S. Lojasiewicza 6, 30-348 Krakow, Poland
}

Correspondence should be addressed to Xianghu Liu; liouxianghu04@126.com

Received 21 April 2013; Revised 18 June 2013; Accepted 27 July 2013

Academic Editor: Naseer Shahzad

Copyright (C) 2013 Xianghu Liu et al. This is an open access article distributed under the Creative Commons Attribution License, which permits unrestricted use, distribution, and reproduction in any medium, provided the original work is properly cited.

This paper is concerned with the existence and uniqueness of mild solution of some fractional impulsive equations. Firstly, we introduce the fractional calculus, Gronwall inequality, and Leray-Schauder's fixed point theorem. Secondly with the help of them, the sufficient condition for the existence and uniqueness of solutions is presented. Finally we give an example to illustrate our main results.

\section{Introduction}

In this paper, we study some fraction evolution with finite impulsive:

$$
\begin{gathered}
{ }^{c} D_{t}^{\alpha} x(t)=A x(t)+I^{1-\alpha} f(t, x(t))+B(t) U(t), \\
t \in J=[0, b], \quad t \neq t_{k}, \\
\Delta x\left(t_{k}\right)=I_{k}\left(x\left(t_{k}^{-}\right)\right) \quad k=1,2,3, \ldots, n, \\
x(0)=x_{0},
\end{gathered}
$$

where ${ }^{c} D_{t}^{\alpha}$ is the standard Caputo fractional derivative of order $\alpha, b>0,0<\alpha<1, A: D(A) \subset X \rightarrow X$ is a generator of a $C_{0}$ semigroup $\{T(t), t \geq 0\}$ defined on a complex Banach space $X$, let $f: J \times X \rightarrow X$ be a given function and satisfying some assumptions that will be specified later, the function $I_{k}: X \rightarrow X$ is continous, and $0=t_{0}<t_{1}<t_{2}<\cdots<t_{k}<$ $\cdots<t_{n}=T, \Delta x\left(t_{k}\right)=x\left(t_{k}^{+}\right)-x\left(t_{k}^{-}\right), x\left(t_{k}^{+}\right)$and $x\left(t_{k}^{-}\right)$denote the right and the left limits of $x(t)$ at $t=t_{k}(k=1,2, \ldots, n), U$ is a given control function in another Banach space $Y$, and $B$ is a linear operator from $Y$ to $X$.

The fractional calculus and fractional difference equations have attracted lots of authors during the past years, and they gave some outstanding work [1-4], because they described many phenomena in engineering, physics, science, and controllability. Delay evolution equation allows someone to think after-effect, so it is a relative important equation. There are some significant development; for example, Wang et al. $[5,6]$ consider the following fractional delay nonlinear integrodiffrential controlled system:

$$
\begin{array}{r}
D_{t}^{q} x(t)+A x(t)=f\left(t, x_{t}, \int_{0}^{t} g\left(t, s, x_{s}\right)\right)+B(t) u(t), \\
t \in I=(0, T], \quad q \in(0,1), \\
x(t)=\phi(t) \quad t \in[-r, 0],
\end{array}
$$

and they used laplace transform and probability density functions to prove some sufficient conditions of some fractional nonlinear finite time delay evolution equations. Shu et al. [7] used the solution operator of semigroup to investigate the system given by

$$
\begin{gathered}
D^{\alpha} x(t)=A x(t)+f(t), \quad \alpha \in(0,1), \\
x(0)=x_{0}, \\
\left.\Delta x\right|_{t=t_{k}}=I_{k}\left(x\left(t_{k}^{-}\right)\right) .
\end{gathered}
$$

Benchohra et al. [8] deal with existence of mild solutions of some fractional functional evolution equations with infinite 
delay. Balachandran et al. [9] concerned the relative controllability of fractional dynamical with delays in control. Specially, Cuevas and Lizama [10] studied some sufficient conditions for the existence and uniqueness of almost automorphic mild solutions to the following semilinear fractional differential equation fractional differential equations:

$$
\begin{gathered}
D^{\alpha} x(t)=A x(t)+D^{\alpha-1} f(t, x(t)), \quad \alpha \in(1,2), \\
x(0)=x_{0} .
\end{gathered}
$$

Bazhlekova [11] studied the fractional evolution equations in Banach spaces. Xue and Xiong [12] concerned the existence and uniqueness of mild solutions for abstract differential equations given by

$$
\begin{gathered}
D_{t}^{q} u(t)=A u(t)+J_{t}^{1-\alpha} f\left(t, u_{t}\right) \quad t \in I=(0, T], \alpha \in(0,1) \\
x(t)=\phi(t) \quad t \in[-r, 0]
\end{gathered}
$$

Motivated by the abovementioned works, we study (1). The rest of this paper is organized as follows. In Section 2, some notation and preparation are given. In Section 3, some mainly results of (1) are obtained. At last, an example is given to demonstrate our results.

\section{Preliminar}

In this section, we will give some definitions and preliminar which will be used in the paper. The norm of the space $X$ will be defined by $\|\cdot\|_{X}$. Let $C(J, X)$ denote the Banach space of all $X$ value continuous functions from $J=[0, T]$ into $X$, the norm $\|\cdot\|_{c}=\sup \|\cdot\|_{X}$. Let the another banach space $P C(J, X)=\{x: J \rightarrow X, x \in$ $C\left(\left(t_{k}, t_{k+1}\right], X\right), k=0,1,2, \ldots, n$, there exist $x\left(t_{k}^{-}\right), x\left(t_{k}^{+}\right)$, $\left.k=1,2, \ldots, n, x\left(t_{k}^{-}\right)=x\left(t_{k}\right)\right\},\|x\|_{P C}=\max \{\sup \| x(t+$ $0) \|$, sup $\|x(t-0)\|\}$. We can use $L^{p}(J, R)$ to denote the Banach space of all Lebesgue measurable functions from $J$ to $R$ with $\|f\|_{L^{p}(J, R)}=\left(\int_{J}|f(t)|^{p} d t\right)^{1 / p}$, and $L^{p}(J, X)$ denote the Banach space of functions $f: J \rightarrow X$ which are Bochner integrable normed by $\|f\|_{L^{p}(J, X)}, u \in L^{p}(J, R)$. [2-4].

Let us recall some known definitions; for more details, see

Let $\alpha, \beta>0$ that $n-1<\alpha<n, n-1<\beta<n$, and $f$ is a suitable function.

Definition 1 (Riemann-Liouville fractional integral and derivative operators). The integral operator $I_{a}^{\alpha}$ is defined on $L_{1}[a$, b] by

$$
I_{a}^{\alpha} f(x)=\frac{1}{\Gamma(\alpha)} \int_{a}^{x}(x-t)^{\alpha-1} f(t) d t, \quad(a \leq x \leq b) .
$$

The derivative operators are defined as $D_{a}^{\alpha} f(x)=$ $D_{a}^{n}\left(I_{a}^{n-\alpha}\right) f(x)$, where $D_{a}^{n}=d^{n} / d t^{n}$ and

$$
I_{a}^{\alpha} I_{a}^{\beta} f(x)=I_{a}^{\alpha+\beta} f(x) .
$$

Definition 2. Caputo fractional derivative of $f(x)$ of order $\alpha$ is defined as

$$
{ }^{c} D_{a}^{\alpha} f(x)=\frac{1}{\Gamma(n-\alpha)} \int_{a}^{x}(x-t)^{n-\alpha-1} f^{(n)}(t) d t .
$$

If $a=0$, we can write the Caputo derivative of the function $f(t) \in C^{n}[0, \infty), f:[0, \infty) \rightarrow R$ via the above RiemannLiouville fractional derivative as

$$
{ }^{c} D_{0}^{\alpha} f(x)={ }^{L} D^{\alpha}\left[f(x)-\sum_{k=0}^{n-1} \frac{x^{k}}{k !} f^{(k)}(0)\right] .
$$

Let us recollect the generalized Gronwall inequality which can be found in [13] and will be used in our main result.

Lemma 3. Suppose $\beta>0, a(t)$ is a nonnegative function locally integrable on $[0, T]$, and $b(t)$ is a nonnegative, nondecreasing continuous function defined on $[0, T], b(t) \leq M$ (constant), and $y(t)$ is nonnegative and locally integrable on $[0, T]$ with

$$
y(t) \leq a(t)+b(t) \int_{0}^{t}(t-s)^{\beta-1} y(s) d s, \quad t \in[0, T] .
$$

Then

$$
\begin{aligned}
y(t) \leq & a(t) \\
& +\int_{0}^{t}\left[\sum_{n=1}^{\infty} \frac{[b(t) \Gamma(\beta)]^{n}}{\Gamma(n \beta)}(t-s)^{n \beta-1} a(s)\right] d s, \\
& t \in[0, T] .
\end{aligned}
$$

Remark 4. Under the hypothesis of Lemma 3, let $a(t)$ be a nondecreasing function on $[0, T]$. Then

$$
y(t) \leq a(t) E_{\beta}\left(b(t) \Gamma(\beta) t^{\beta}\right)
$$

where $E_{\beta}$ is the Mittag-Leffler function defined by

$$
E_{\beta}(z)=\sum_{k=0}^{\infty} \frac{z^{k}}{\Gamma(k \beta+1)} .
$$

By Lemma 3 and Remark 4, we can establish a useful nonlinear impulsive Gronwall inequality which will be used in calculating.

Lemma 5 (see [14]). Let $x \in P C(J, X)$ satisfy the following inequality:

$$
\|x(t)\| \leq c_{1}+c_{2} \int_{0}^{t}(t-s)^{\beta-1}\|x(s)\| d s+\sum_{0<t_{k}<t} h_{k}\left\|x\left(t_{k}^{-}\right)\right\|,
$$

where $c_{1}, c_{2}, h_{k} \geq 0$ are constants. Then

$$
\begin{aligned}
\|x(t)\| \leq & c_{1}\left(1+H^{*} E_{\beta}\left(c_{2} \Gamma(\beta) t^{\beta}\right)\right)^{k} \\
& \times E_{\beta}\left(c_{2} \Gamma(\beta) t^{\beta}\right) \quad \text { for } t \in\left(t_{k}, t_{k+1}\right],
\end{aligned}
$$

where $H^{*}=\max \left\{h_{k}: k=1,2, \ldots, m\right\}$. 
Specially, if $\beta=1$,

$$
\|x(t)\| \leq c_{1}\left(1+H^{*} E_{1}\left(c_{2} t\right)^{k}\right) E_{1}\left(c_{2} t\right) \quad \text { for } t \in\left(t_{k}, t_{k+1}\right] .
$$

We also introduce the following theorem that will be used in our mainly result.

Theorem 6 (Hölder's inequality). Assume that $p>0, q>0$, and $1 / p+1 / q=1$; if $f \in L^{p}(\Omega)$ and $g \in L^{q}(\Omega)$ then $f \cdot g \in$ $L^{1}(\Omega)$ and $\|f g\|_{L^{1}(\Omega)} \leq\|f\|_{L^{p}(\Omega)}\|g\|_{L^{q}(\Omega)}$.

Theorem 7 (Arzela-Ascoli theorem). If a sequence $\left(f_{n}\right)$ in $C(x)$ is bounded and equicontinuous, then it has a uniformly convergent subsequence.

Theorem 8 (Leray-Schauder's fixed point theorem). If $C$ is a closed bounded and convex subset of Banach space $X$ and $F$ : $C \rightarrow C$ is completely continuous, then the $F$ has a fixed point in $C$.

\section{Existence and Uniqueness of Mild Solution}

In this section, we will investigate the existence and uniqueness for impulsive fractional differential equations with the help of the Leray-Schauder's fixed point theorem and someone else. Without loss of generality, let $t \in\left(t_{k}, t_{k+1}\right], 1 \leq k \leq$ $n-1$.

Firstly, we will make the following assumptions be satisfied on the data of our problem.

$H(1):\{T(t), t>0\}$ is a compact semigroup, and there exists a constant $M>0$, such that $M=$ $\sup _{t \in[0, \infty)}\|T(t)\|_{L_{b}(X)}<\infty$.

$H(2)$ : The function $f: J \times X \rightarrow X$ satisfies the following:

(i) $f$ is measurable for all $t \in J$;

(ii) there exists a constant $L_{f}>0$ such that $\| f(t, x)-$ $f(t, y)\left\|\leq L_{f}\right\| x-y \|$, for all $x, y \in X$;

(iii) there exists a real function $\phi(t) \in L^{1 / \gamma}\left(J, R^{+}\right), \gamma \in$ $(0, \alpha)$, and a constant $\theta>0$, such that $\|f(t, x)\| \leq$ $\phi(t)+\theta\|x\|$, for a.e. $t>0$ and all $x \in X$.

$H(3): I_{i}: X \rightarrow X(i=1,2, \ldots, n)$ satisfies the following:

(i) $I_{i}$ maps a bounded set to a bounded set;

(ii) there exist constants $h_{i}>0(i=1,2, \ldots, n)$ such that

$$
\left\|I_{i}(x)-I_{i}(y)\right\| \leq h_{i}\|x-y\|, \quad x, y \in X
$$

(iii) $\|I(0)\|=\max \left(\left\|I_{1}(0)\right\|,\left\|I_{2}(0)\right\|, \ldots,\left\|I_{n}(0)\right\|\right)$.

$H(4)$ : Let $Y$ be a separable reflexive Banach space. Operator $B \in L^{\infty}(J, L(Y, X)),\|B\|_{\infty}$, stands for the norm of operator $B$ on Banach space $L^{\infty}(J, L(Y, X))$.

$H(5):$ The multivalued maps $U: J \rightarrow P_{f}(Y)$ (where $P_{f}(Y)$ is a class of nonempty closed and convex subsets of $Y$ ) are measurable and $U(\cdot) \subseteq \Omega$ where $\Omega$ are a bounded set of $Y$.
Set the admissible control set:

$$
U_{a d}=S_{U}^{p}=\left\{u \in L^{p}(\Omega): u(t) \in U(t) \text { a.e. }\right\}, \quad 1<p<\infty .
$$

Then, $U_{a d} \neq \emptyset$ (see Proposition 2.1.7 and Lemma 2.3.2 of [15]). And it is obvious that $B u \in L^{p}(J, X)$ for all $u \in U_{a d}$.

According to Definitions 1 and 2 and by comparison with the fractional differential equations given in $[5,16,17]$, then we shall define the concept of mild solution for problem (1) as follows.

Definition 9. A function $x \in P C(J, X)$ is said to be a solution (mild solution) of the problem (1) if $x(0)=x_{0}$ such that

$$
\begin{aligned}
x(t)= & S_{\alpha}(t) x_{0}+\sum_{i=1}^{k} S_{\alpha}\left(t-t_{i}\right) I_{i}\left(x\left(t_{i}^{-}\right)\right) \\
& +\int_{0}^{t} S_{\alpha}(t-s) f(s, x(s)) d s \\
& +\int_{0}^{t}(t-s)^{\alpha-1} T_{\alpha}(t-s) B(s) u(s) d s,
\end{aligned}
$$

where

$$
\begin{gathered}
S_{\alpha}(t)=\int_{0}^{\infty} \xi_{\alpha}(\theta) T\left(t^{\alpha} \theta\right) d \theta, \\
T_{\alpha}(t)=\alpha \int_{0}^{\infty} \theta \xi_{\alpha}(\theta) T\left(t^{\alpha} \theta\right) d \theta, \\
\xi_{\alpha}(\theta)=\frac{1}{\alpha} \theta^{-1-1 / \alpha} ळ_{\alpha}\left(\theta^{-1 / \alpha}\right) \geq 0, \\
\omega_{\alpha}(\theta)=\frac{1}{\pi} \sum_{n=1}^{\infty}(-1)^{n-1} \theta^{-n \alpha-1} \frac{\Gamma(n \alpha+1)}{n !} \sin (n \pi \alpha), \\
\theta \in(0, \infty),
\end{gathered}
$$

where $\xi_{\alpha}$ is a probability density function defined on $(0, \infty)$, that is

$$
\xi_{\alpha}(\theta) \geq 0, \quad \theta \in(0, \infty), \quad \int_{0}^{\infty} \xi_{\alpha}(\theta)=1 .
$$

Lemma 10 (see [17]). The operators $S_{\alpha}(t)$ and $T_{\alpha}(t)$ have the following properties and there exists $M$ as described in $H(1)$.

(i) For any fixed $t \geq 0, S_{\alpha}(t)$ and $T_{\alpha}(t)$ are linear and bounded operators; that is, for any $x \in X$,

$$
\left\|S_{\alpha}(t) x\right\| \leq M\|x\|, \quad\left\|T_{\alpha}(t) x\right\| \leq \frac{M}{\Gamma(\alpha)}\|x\| .
$$

(ii) $\left\{S_{\alpha}(t), t \geq 0\right\}$ and $\left\{T_{\alpha}(t), t \geq 0\right\}$ are strongly continuous.

(iii) For any $t \geq 0, S_{\alpha}(t)$ and $T_{\alpha}(t)$ are also compact operators if $T(t)$ is compact.

Lemma 11. If the assumptions $H(1)-H(4)$ are satisfied and (1) is mildly solvable on $[0, b]$, then there exists a constant $\omega>0$ such that $\|x(t)\| \leq \omega$. 
Proof. If (1) can be solvable on $[0, b]$, we may suppose $x(t)$ is the mild solution of it, so $x(t)$ must satisfy (19) as follows:

$$
\begin{aligned}
x(t)= & S_{\alpha}(t) x_{0}+\sum_{i=1}^{k} S_{\alpha}\left(t-t_{i}\right) I_{i}\left(x\left(t_{i}^{-}\right)\right) \\
& +\int_{0}^{t} S_{\alpha}(t-s) f(s, x(s)) d s \\
& +\int_{0}^{t}(t-s)^{\alpha-1} T_{\alpha}(t-s) B(s) u(s) d s .
\end{aligned}
$$

For $t \in\left(t_{k}, t_{k+1}\right], 1 \leq k \leq n-1$, through calculating, we can get that

$$
\begin{aligned}
\|x(t)\| \leq & \left\|S_{\alpha}(t) x_{0}\right\|+\left\|\sum_{i=1}^{k} S_{\alpha}\left(t-t_{i}\right) I_{i}\left(x\left(t_{i}^{-}\right)\right)\right\| \\
& +\int_{0}^{t}\left\|S_{\alpha}(t-s) f(s, x(s))\right\| d s \\
& +\int_{0}^{t}(t-s)^{\alpha-1}\left\|T_{\alpha}(t-s) B(s) u(s)\right\| d s \\
\leq & M\left\|x_{0}\right\|+M \sum_{i=1}^{n} h_{i}\left\|x\left(t_{i}^{-}\right)\right\|+M n\|I(0)\| \\
& +M \int_{0}^{t}[\phi(s)+\theta\|x(s)\|] d s \\
& +\frac{M\|B\|_{\infty}}{\Gamma(\alpha)} \int_{0}^{t}(t-s)^{\alpha-1}\|u(s)\| d s \\
& +\frac{M\|B\|_{\infty}}{\Gamma(\alpha)}\left(\frac{p-1}{p \alpha-1}\right)^{(p-1) / p} b^{\alpha-1 / p}\|u\|_{L^{p}} \\
& +M \theta \int_{0}^{t}\|x(s)\| d s \\
& +M n\|I(0)\|+M \sum_{i=1}^{n} h_{i}\left\|x\left(t_{i}^{-}\right)\right\| \\
& \\
& =\phi \|_{L^{1 / \gamma}} \\
& \\
& \\
& \\
& \\
&
\end{aligned}
$$

Let $\rho=M\left\|x_{0}\right\|+M n\|I(0)\|+M b^{1-r}\|\phi\|_{L^{1 / \gamma}}+\left(M\|B\|_{\infty} /\right.$ $\Gamma(\alpha))(p-1 / p \alpha-1)^{(p-1) / p} b^{\alpha-1 / p}\|u\|_{L^{p}}$, then

$$
\|x(t)\| \leq \rho+M \sum_{i=1}^{n} h_{i}\left\|x\left(t_{i}^{-}\right)\right\|+M \theta \int_{0}^{t}\|x(s)\| d s,
$$

so it follows from Lemma 5,

$$
\|x(t)\| \leq \rho\left(1+H^{*} E_{1}(M \theta b)\right)^{k} E_{1}(M \theta b)=\omega
$$

where

$$
H^{*}=\max \left\{M h_{i}: i=1,2, \ldots, n\right\} .
$$

The proof is completed.
Theorem 12. Assume that the hypotheses $H(1)-H(4)$ are satisfied, and then the problem (1) has an unique mild solution on J provided that

$$
\left(\sum_{i=1}^{n} h_{i}+\theta b\right) M<1
$$

Proof. Transform the problem (1) into a fixed point theorem. Consider the operator $F: P C(J, X) \rightarrow P C(J, X)$ defined by

$$
\begin{aligned}
(F x)(t)= & S_{\alpha}(t) x_{0}+\sum_{i=1}^{k} S_{\alpha}\left(t-t_{i}\right) I_{i}\left(x\left(t_{i}^{-}\right)\right) \\
& +\int_{0}^{t} S_{\alpha}(t-s) f(s, x(s)) d s \\
& +\int_{0}^{t}(t-s)^{\alpha-1} T_{\alpha}(t-s) B(s) u(s) d s .
\end{aligned}
$$

Clearly, the problem of finding mild solutions of (1) is reduced to find the fixed points of the $F$, the proof base on Theorem 8 . Now we prove that the operator $F$ satisfies all the conditions of the Theorem 8 .

Firstly, choose

$$
\begin{aligned}
M[\| & x_{0}\|+n\| I(0)\left\|+b^{1-r}\right\| \phi \|_{L^{1 / \gamma}} \\
+ & \left.\frac{\|B\|_{\infty}}{\Gamma(\alpha)}\left(\frac{p-1}{p \alpha-1}\right)^{(p-1) / p} b^{\alpha-1 / p}\|u\|_{L^{p}}\right] \\
& \times\left(1-M \sum_{i=1}^{n} h_{i}-M b \theta\right)^{-1} \leq r,
\end{aligned}
$$

and consider the bounded set $B_{r}=\{x \in P C:\|x\| \leq r\}$.

Next, for the sake of convenient, we divide the proof into several steps.

Step 1. We prove that $F B_{r} \subseteq B_{r}$.

In fact, for each $x \in B_{r}, t \in\left(t_{k}, t_{k+1}\right], 1 \leq k \leq n-1$, we have

$$
\begin{aligned}
\|(F x)(t)\| \leq & \left\|S_{\alpha}(t) x_{0}\right\|+\left\|\sum_{i=1}^{k} S_{\alpha}\left(t-t_{i}\right) I_{i}\left(x\left(t_{i}^{-}\right)\right)\right\| \\
& +\int_{0}^{t}\left\|S_{\alpha}(t-s) f(s, x(s))\right\| d s \\
& +\int_{0}^{t}(t-s)^{\alpha-1}\left\|T_{\alpha}(t-s) B(s) u(s)\right\| d s \\
\leq & M\left\|x_{0}\right\|+M \sum_{i=1}^{n} h_{i}\left\|x\left(t_{i}^{-}\right)\right\|+M n\|I(0)\| \\
& +M \int_{0}^{t}[\phi(s)+\theta\|x(s)\|] d s \\
& +\frac{M\|B\|_{\infty}}{\Gamma(\alpha)} \int_{0}^{t}(t-s)^{\alpha-1}\|u(s)\| d s
\end{aligned}
$$




$$
\begin{aligned}
\leq & M\left\|x_{0}\right\|+M \sum_{i=1}^{n} h_{i}\left\|x\left(t_{i}^{-}\right)\right\| \\
& +M n\|I(0)\|+M b^{1-\gamma}\|\phi\|_{L^{1 / \gamma}} \\
& +M \theta \int_{0}^{t}\|x(s)\| d s \\
& +\frac{M\|B\|_{\infty}}{\Gamma(\alpha)}\left(\frac{p-1}{p \alpha-1}\right)^{(p-1) / p} b^{\alpha-(1 / p)}\|u\|_{L^{p}} \\
\leq & M\left\|x_{0}\right\|+M n\|I(0)\| \\
& +M b^{1-\gamma}\|\phi\|_{L^{1 / \gamma}}+\frac{M\|B\|_{\infty}}{\Gamma(\alpha)}\left(\frac{p-1}{p \alpha-1}\right)^{(p-1) / p} \\
& \times b^{\alpha-(1 / p)}\|u\|_{L^{p}} \\
& +\left(M \sum_{i=1}^{n} h_{i}+M b \theta\right) r \\
\leq & r .
\end{aligned}
$$

Hence, we can deduce that $F B_{r} \subseteq B_{r}$.

Step 2. We show that $F$ is continuous.

Let $\left\{x_{n}\right\}$ be a sequence such that $x_{n} \rightarrow x$ in $P C(J, X)$ as $n \rightarrow \infty$. Then, for each $t \in\left(t_{k}, t_{k+1}\right], 1 \leq k \leq n-1$, we obtain

$$
\begin{aligned}
& \left\|\left(F x_{n}\right)(t)-(F x)(t)\right\| \\
& \leq\left\|\sum_{i=1}^{k} S_{\alpha}\left(t-t_{i}\right)\left[I_{i}\left(x_{n}\left(t_{i}^{-}\right)\right)-I_{i}\left(x\left(t_{i}^{-}\right)\right)\right]\right\| \\
& \quad+\int_{0}^{t}\left\|S_{\alpha}(s)\left[f\left(s, x_{n}(s)\right)-f(s, x(s))\right]\right\| d s \\
& \leq M \sum_{i=1}^{n} h_{i}\left\|x_{n}-x\right\|+M L_{f} \int_{0}^{t}\left\|x_{n}(s)-x(s)\right\| d s \\
& \leq\left[M \sum_{i=1}^{n} h_{i}+M L L_{f} b\right]\left\|x_{n}-x\right\|,
\end{aligned}
$$

as $x_{n} \rightarrow x$, and it is easy to see that

$$
\left\|F x_{n}-F x\right\| \longrightarrow \text { as } n \longrightarrow \infty
$$

that is, $F$ is continuous.

Step 3. $F$ is equicontinuous on $B_{r}$.

Let $0 \leq \tau_{1}<\tau_{2} \leq b$; then, for each $x \in B_{r}$, we obtain

$$
\begin{aligned}
& \left\|(F x)\left(\tau_{2}\right)-(F x)\left(\tau_{1}\right)\right\| \\
& \leq\left\|\left[S_{\alpha}\left(\tau_{2}\right)-S_{\alpha}\left(\tau_{1}\right)\right] x_{0}\right\|
\end{aligned}
$$

$$
\begin{aligned}
& +\left\|\left[\sum_{i=1}^{k} S_{\alpha}\left(\tau_{2}-t_{i}\right)-\sum_{i=1}^{k} S_{\alpha}\left(\tau_{1}-t_{i}\right)\right] I_{i}\left(x\left(t_{i}^{-}\right)\right)\right\| \\
& +\| \int_{0}^{\tau_{2}} S_{\alpha}\left(\tau_{2}-s\right) f(s, x(s)) d s \\
& -\int_{0}^{\tau_{1}} S_{\alpha}\left(\tau_{1}-s\right) f(s, x(s)) d s \| \\
& +\| \int_{0}^{\tau_{2}}\left(\tau_{2}-s\right)^{\alpha-1} T_{\alpha}\left(\tau_{2}-s\right) B(s) u(s) d s \\
& -\int_{0}^{\tau_{1}}\left(\tau_{1}-s\right)^{\alpha-1} T_{\alpha}\left(\tau_{1}-s\right) B(s) u(s) d s \| \\
& \leq\left\|S_{\alpha}\left(\tau_{2}\right)-S_{\alpha}\left(\tau_{1}\right)\right\|\left\|x_{0}\right\| \\
& +\sum_{i=1}^{k}\left\|S_{\alpha}\left(\tau_{2}-t_{i}\right)-S_{\alpha}\left(\tau_{1}-t_{i}\right)\right\| \\
& \times\left(h_{i}\left\|x\left(t_{i}^{-}\right)\right\|+\left\|I_{i}(0)\right\|\right) \\
& +\left\|\int_{0}^{\tau_{1}}\left(S_{\alpha}\left(\tau_{2}-s\right)-S_{\alpha}\left(\tau_{1}-s\right)\right) f(s, x(s)) d s\right\| \\
& +\left\|\int_{\tau_{1}}^{\tau_{2}} S_{\alpha}\left(\tau_{2}-s\right) f(s, x(s)) d s\right\| \quad \text { denoted by } Q_{1} \\
& +\left\|\int_{\tau_{1}}^{\tau_{2}}\left(\tau_{2}-s\right)^{\alpha-1} T_{\alpha}\left(\tau_{2}-s\right) B(s) u(s) d s\right\|
\end{aligned}
$$

denoted by $Q_{2}$

$+\| \int_{0}^{\tau_{1}}\left[\left(\tau_{2}-s\right)^{\alpha-1}-\left(\tau_{1}-s\right)^{\alpha-1}\right]$

$$
\times T_{\alpha}\left(\tau_{2}-s\right) B(s) u(s) d s \| \quad \text { denoted by } Q_{3}
$$$$
+\| \int_{0}^{\tau_{1}}\left(\tau_{1}-s\right)^{\alpha-1}\left[T_{\alpha}\left(\tau_{2}-s\right)-T_{\alpha}\left(\tau_{1}-s\right)\right]
$$

$$
\times B(s) u(s) d s \|
$$

denoted by $Q_{4}$.

Let

$$
\begin{aligned}
\Lambda= & \left\|S_{\alpha}\left(\tau_{2}\right)-S_{\alpha}\left(\tau_{1}\right)\right\|\left(\left\|x_{0}\right\|+N\right) \\
& +\sum_{i=1}^{k}\left\|S_{\alpha}\left(\tau_{2}-t_{i}\right)-S_{\alpha}\left(\tau_{1}-t_{i}\right)\right\|\left(h_{i}\left\|x\left(t_{i}^{-}\right)\right\|+\left\|I_{i}(0)\right\|\right) \\
& +\left\|\int_{0}^{\tau_{1}}\left(S_{\alpha}\left(\tau_{2}-s\right)-S_{\alpha}\left(\tau_{1}-s\right)\right) f(s, x(s)) d s\right\| .
\end{aligned}
$$

By (ii) of Lemma 10, we have

$$
\lim _{\tau_{2} \rightarrow \tau_{1}} \Lambda=0
$$


By the assumption $H(2)$, we obtain

$$
Q_{1} \leq M\left(\|\phi\|_{L^{1 / \gamma}}+\theta b^{\gamma} r\right)\left(\tau_{2}-\tau_{1}\right)^{1-\gamma}
$$

and we get

$$
\begin{aligned}
Q_{2} \leq & \frac{M\|B\|_{\infty}}{\Gamma(\alpha)}\left(\frac{p-1}{p \alpha-1}\right)^{(p-1) / p}\|u\|_{L^{p}}\left(\tau_{2}-\tau_{1}\right)^{\alpha-(1 / p)} \\
Q_{3} \leq & \frac{2 M\|B\|_{\infty}}{\Gamma(\alpha)}\left(\frac{p-1}{p \alpha-1}\right)^{(p-1) / p}\|u\|_{L^{p}}\left(\tau_{2}-\tau_{1}\right)^{\alpha-(1 / p)} \\
Q_{4} \leq & \sup _{s \in\left[0, \tau_{1}-\varepsilon\right]}\left\|T_{\alpha}\left(\tau_{2}-s\right)-T_{\alpha}\left(\tau_{1}-s\right)\right\| \\
& \times\left(\frac{p-1}{p \alpha-1}\right)^{(p-1) / p}\left(\tau_{1}^{(p \alpha-1) /(p-1)}-\varepsilon^{(p \alpha-1) /(p-1)}\right)^{(p-1) / p} \\
& \times\|B\|_{\infty}\|u\|_{L^{p}} \\
& +\frac{2 M\|B\|_{\infty}}{\Gamma(\alpha)}\left(\frac{p-1}{p \alpha-1}\right)^{(p-1) / p}\|u\|_{L^{p}} \varepsilon^{\alpha-(1 / p)} .
\end{aligned}
$$

Combining the estimations for $\Lambda, Q_{i}(i=1, \ldots, 4)$, let $\tau_{2} \rightarrow \tau_{1}$ and $\varepsilon \rightarrow 0$, and we know that $\|(F x)\left(\tau_{2}\right)-$ $(F x)\left(\tau_{1}\right) \| \rightarrow 0$, which implies that $F$ is equicontinuous.

Step 4. Now we show that $F$ is compact.

Let $t \in\left(t_{k}, t_{k+1}\right], 1 \leq k \leq n-1$ be fixed, and we show that the set $\Pi(t)=\left\{(F x)(t): x \in B_{r}\right\}$ is relatively compact in $X$.

Clearly, $\Pi(0)=\left\{x_{0}-g(x)\right\}$ is compact, so it is only necessary to consider $t>0$. For each $\epsilon \in(0, t), t \in(0, b]$, $x \in B_{r}$ and any $\delta>0$, we define

$$
\Pi_{\epsilon, \delta}(t)=\left\{F_{\epsilon, \delta}(x)(t): x \in B_{r}\right\},
$$

where

$$
\begin{aligned}
F_{\epsilon, \delta}(x)(t)= & S_{\alpha}(t) x_{0}+\sum_{i=1}^{k} S_{\alpha}\left(t-t_{i}\right) I_{i}\left(x\left(t_{i}^{-}\right)\right) \\
& +\int_{0}^{t-\epsilon} \int_{\delta}^{\infty} \xi_{\alpha}(\theta) T\left((t-s)^{\alpha} \theta\right) f(s) d \theta d s \\
& +\alpha \int_{0}^{t-\epsilon}(t-s)^{\alpha-1} \\
& \times \int_{\delta}^{\infty} \theta \xi_{\alpha}(\theta) T\left((t-s)^{\alpha} \theta\right) B(s) u(s) d \theta d s \\
= & S_{\alpha}(t) x_{0}+\sum_{i=1}^{k} S_{\alpha}\left(t-t_{i}\right) I_{i}\left(x\left(t_{i}^{-}\right)\right) \\
& +T\left(\epsilon^{\alpha} \delta\right) \\
& \times \int_{0}^{t-\epsilon} \int_{\delta}^{\infty} \xi_{\alpha}(\theta) T\left((t-s)^{\alpha} \theta-\epsilon^{\alpha} \delta\right) f(s) d \theta d s
\end{aligned}
$$

$$
\begin{aligned}
+\alpha T\left(\epsilon^{\alpha} \delta\right) \int_{0}^{t-\epsilon} \int_{\delta}^{\infty} & \theta(t-s)^{\alpha-1} \xi_{\alpha}(\theta) T \\
& \times\left((t-s)^{\alpha} \theta-\epsilon^{\alpha} \delta\right) \\
& \times B(s) u(s) d \theta d s .
\end{aligned}
$$

From the compactness of $T\left(\epsilon^{\alpha} \delta\right)\left(\epsilon^{\delta}>0\right)$, we obtain that the set $\Pi_{\epsilon, \delta}(t)=\left\{F_{\epsilon, \delta}(x)(t): x \in B_{r}\right\}$ is relatively compact set in $X$ for each $\epsilon \in(0, t)$ and $\delta>0$. Moreover, we have

$$
\begin{aligned}
& \left\|F(x)(t)-F_{\epsilon, \delta}(x)(t)\right\| \\
& =\| \int_{0}^{t} \int_{0}^{\infty} \xi_{\alpha}(\theta) T\left((t-s)^{\alpha} \theta\right) f(s) d \theta d s \\
& -\int_{0}^{t-\epsilon} \int_{\delta}^{\infty} \xi_{\alpha}(\theta) T\left((t-s)^{\alpha} \theta\right) f(s) d \theta d s \| \\
& +\| \alpha \int_{0}^{t} \int_{0}^{\infty} \theta(t-s)^{\alpha-1} \xi_{\alpha}(\theta) \\
& \times T\left((t-s)^{\alpha} \theta\right) B(s) u(s) d \theta d s \\
& -\alpha \int_{0}^{t-\epsilon} \int_{\delta}^{\infty} \theta(t-s)^{\alpha-1} \xi_{\alpha}(\theta) T\left((t-s)^{\alpha} \theta\right) \\
& \times B(s) u(s) d \theta d s \| \\
& \leq\left\|\int_{0}^{t} \int_{0}^{\delta} \xi_{\alpha}(\theta) T\left(s^{\alpha} \theta\right) f(s) d \theta d s\right\| \\
& +\left\|\int_{t-\epsilon}^{t} \int_{\delta}^{\infty} \xi_{\alpha}(\theta) T\left(s^{\alpha} \theta\right) f(s) d \theta d s\right\| \\
& +\alpha \| \int_{0}^{t} \int_{0}^{\delta} \theta(t-s)^{\alpha-1} \xi_{\alpha}(\theta) \\
& \times T\left((t-s)^{\alpha} \theta\right) B(s) u(s) d \theta d s \| \\
& +\alpha \| \int_{t-\epsilon}^{t} \int_{\delta}^{\infty} \theta(t-s)^{\alpha-1} \xi_{\alpha}(\theta) \\
& \times T\left((t-s)^{\alpha} \theta\right) B(s) u(s) d \theta d s \| \\
& \leq M\left(\left\|b^{1-\gamma} \phi\right\|_{L(1 / \gamma)}+\theta r b\right) \int_{0}^{\delta} \xi_{\alpha}(\theta) d \theta \\
& +M\left(\epsilon^{1-\gamma}\|\phi\|_{L(1 / \gamma)}+\theta r \epsilon\right) \int_{\delta}^{\infty} \xi_{\alpha}(\theta) d \theta \\
& +\frac{\alpha M\|B\|_{\infty}}{\Gamma(\alpha)}\left(\frac{p-1}{p \alpha-1}\right)^{(p-1) / p}\|u\|_{L^{p}} b^{\alpha-1 / p} \\
& \times \int_{0}^{\delta} \theta \xi_{\alpha}(\theta) d \theta
\end{aligned}
$$




$$
\begin{aligned}
& +\frac{\alpha M\|B\|_{\infty}}{\Gamma(\alpha)}\left(\frac{p-1}{p \alpha-1}\right)^{(p-1) / p}\|u\|_{L^{p}} \epsilon^{\alpha-1 / p} \\
& \times \int_{\delta}^{\infty} \theta \xi_{\alpha}(\theta) d \theta
\end{aligned}
$$

when $\epsilon \rightarrow 0$ and $\delta \rightarrow 0$, we can easily find (1) $\rightarrow 0$, $(2) \rightarrow 0$, (3) $\rightarrow 0$, (4) $\rightarrow 0$. Therefore, there are relatively compact sets arbitrarily close to the set $\Pi(t), t>0$. Hence the set $\Pi(t), t>0$ is also relatively compact in $X$.

As a result, by the conclusion of Theorem 8 , we obtain that $F$ has a fixed point $x$ on $B_{r}$. So system (1) has a unique mild solution on $J$. The proof is completed.

\section{Optimal Control Results}

In the following, we will consider the Lagrange problem (P).

Find a control pair $\left(x^{0}, u^{0}\right) \in P C(J, X) \times U_{a d}$ such that

$$
\mathscr{J}\left(x^{0}, u^{0}\right) \leq J\left(x^{u}, u\right), \quad \forall(x, u) \in P C(J, X) \times U_{a d},
$$

where

$$
\mathscr{J}\left(x^{u}, u\right):=\int_{0}^{b} \mathscr{L}\left(t, x^{u}(t), u(t)\right) d t
$$

and $x^{u}$ denotes the mild solution of system (1) corresponding to the control $u \in U_{a d}$.

For the existence of solution for problem (P), we shall introduce the following assumption.

$H(6)$ : The function $\mathscr{L}: J \times X \times Y \rightarrow R \cup\{\infty\}$ satisfies the following.

(i) The function $\mathscr{L}: J \times X \times Y \rightarrow R \cup\{\infty\}$ is Borel measurable;

(ii) $\mathscr{L}(t, \cdot, \cdot)$ is sequentially lower semicontinuous on $X \times$ $Y$ for almost all $t \in J$;

(iii) $\mathscr{L}(t, x, \cdot)$ is convex on $Y$ for each $x \in X$ and almost all $t \in J$;

(iv) there exist constants $c \geq 0, d>0, \varphi$ is nonnegative, and $\varphi \in L^{1}(J, R)$ such that

$$
\mathscr{L}(t, x, u) \geq \varphi(t)+c\|x\|_{X}+d\|u\|_{Y}^{p} .
$$

Next, we can give the following result on existence of optimal controls for problem (P).

Theorem 13. Let the assumptions of Theorem 12 and $H(6)$ hold. Suppose that $B$ is a strongly continuous operator. Then Lagrange problem $(P)$ admits at least one optimal pair; that is, there exists an admissible control pair $\left(x^{0}, u^{0}\right) \in P C(J, X) \times$ $U_{a d}$ such that

$$
\begin{array}{r}
\mathscr{J}\left(x^{0}, u^{0}\right)=\int_{0}^{b} \mathscr{L}\left(t, x^{0}(t), u^{0}(t)\right) d t \leq \mathscr{J}\left(x^{u}, u\right), \\
\forall\left(x^{u}, u\right) \in P C(J, X) \times U_{a d} .
\end{array}
$$

Proof. If $\inf \left\{\mathscr{J}\left(x^{u}, u\right):\left(x^{u}, u\right) \in P C(J, X) \times U_{a d}\right\}=+\infty$, there is nothing to prove.

Without loss of generality, we assume that $\inf \left\{J\left(x^{u}, u\right)\right.$ : $\left.\left(x^{u}, u\right) \in P C(J, X) \times U_{a d}\right\}=\rho<+\infty$. Using $H(6)$, we have $\rho>-\infty$. By definition of infimum, there exists a minimizing sequence feasible pair $\left\{\left(x^{m}, u^{m}\right)\right\} \subset \mathscr{P}_{a d} \equiv\{(x, u): x$ is a mild solution of system (31) corresponding to $\left.u \in U_{a d}\right\}$, such that $J\left(x^{m}, u^{m}\right) \rightarrow \rho$ as $m \rightarrow+\infty$. Since $\left\{u^{m}\right\} \subseteq U_{a d}, m=$ $1,2, \ldots,\left\{u^{m}\right\}$ is a bounded subset of the separable reflexive Banach space $L^{p}(J, Y)$, there exists a subsequence, relabeled as $\left\{u^{m}\right\}$, and $u^{0} \in L^{p}(J, Y)$ such that

$$
u^{m} \stackrel{w}{\longrightarrow} u^{0} \quad \text { in } L^{p}(J, Y) .
$$

Since $U_{a d}$ is closed and convex, due to Marzur lemma, $u_{0} \in U_{a d}$. Let $\left\{x^{m}\right\}$ denote the sequence of solutions of the system (1) corresponding to $\left\{u^{m}\right\}, x^{0}$ is the mild solution of the system (1) corresponding to $u^{0} \cdot x^{m}$ and $x^{0}$ satisfy the following integral equation, respectively:

$$
\begin{aligned}
x^{m}(t)= & S_{\alpha}(t) x_{0}+\sum_{i=1}^{k} S_{\alpha}\left(t-t_{i}\right) I_{i}\left(x^{m}\left(t_{i}^{-}\right)\right) \\
& +\int_{0}^{t} S_{\alpha}(t-s) f\left(s, x^{m}(s)\right) d s \\
& +\int_{0}^{t}(t-s)^{\alpha-1} T_{\alpha}(t-s) B(s) u^{m}(s) d s, \\
x^{0}(t)= & S_{\alpha}(t) x_{0}+\sum_{i=1}^{k} S_{\alpha}\left(t-t_{i}\right) I_{i}\left(x^{0}\left(t_{i}^{-}\right)\right) \\
& +\int_{0}^{t} S_{\alpha}(t-s) f\left(s, x^{0}(s)\right) d s \\
& +\int_{0}^{t}(t-s)^{\alpha-1} T_{\alpha}(t-s) B(s) u^{0}(s) d s .
\end{aligned}
$$

It follows the boundedness of $\left\{u^{m}\right\},\left\{u^{0}\right\}$ and Lemma 11, one can check that there exists a positive number $\omega$ such that $\left\|x^{m}\right\| \leq \omega,\left\|x^{0}\right\| \leq \omega$.

For $t \in J$, we obtain

$$
\begin{aligned}
& \left\|x^{m}(t)-x^{0}(t)\right\| \\
& =\left\|\sum_{i=1}^{k} S_{\alpha}\left(t-t_{i}\right)\left[I_{i}\left(x^{m}\left(t_{i}^{-}\right)\right)-I_{i}\left(x^{0}\left(t_{i}^{-}\right)\right)\right]\right\| \\
& \quad \text { denoted by } \eta_{1}(t) \\
& +\int_{0}^{t} \| S_{\alpha}(t-s)\left[\left[f\left(s, x^{m}(s)\right)-f\left(s, x^{0}(s)\right)\right] \| d s\right. \\
& +\int_{0}^{t}(t-s)^{\alpha-1} \\
& \quad \times\left\|T_{\alpha}(t-s)\left[B(s) u^{m}(s)-B(s) u^{0}(s)\right]\right\| d s, \\
& \quad \text { denoted by } \eta_{2}(t)
\end{aligned}
$$


By $H(3)(i i)$, we have

$$
\begin{aligned}
\eta_{1}(t) & =\left\|\sum_{i=1}^{k} S_{\alpha}\left(t-t_{i}\right)\left[I_{i}\left(x^{m}\left(t_{i}^{-}\right)\right)-I_{i}\left(x^{0}\left(t_{i}^{-}\right)\right)\right]\right\| \\
& \leq M \sum_{i=1}^{n} h_{i}\left\|x^{m}-x^{0}\right\| \longrightarrow 0, \quad \text { as } m \longrightarrow \infty .
\end{aligned}
$$

Using Lemma 10(i) and by $H(2)(\mathrm{ii})$, one can obtain

$$
\begin{aligned}
\eta_{2}(t) & =\int_{0}^{t}\left\|S_{\alpha}(t-s)\left[f\left(s, x^{m}(s)\right)-f\left(s, x^{0}(s)\right)\right]\right\| d s \\
& \leq M L_{f} \int_{0}^{t}\left\|x^{m}(s)-x^{0}(s)\right\| d s .
\end{aligned}
$$

Similarly, one has

$$
\begin{aligned}
\eta_{3}(t)= & \int_{0}^{t}(t-s)^{\alpha-1} \\
& \times\left\|T_{\alpha}(t-s)\left[B(s) u^{m}(s)-B(s) u^{0}(s)\right]\right\| d s \\
\leq & \frac{M}{\Gamma(\alpha)}\left(\frac{p-1}{p \alpha-1}\right)^{(p-1) / p} t^{\alpha-(1 / p)} \\
& \times\left(\int_{0}^{t}\left\|B(s) u^{m}(s)-B(s) u^{0}(s)\right\|^{p} d s\right)^{1 / p} \\
\leq & \frac{M}{\Gamma(\alpha)}\left(\frac{p-1}{p \alpha-1}\right)^{(p-1) / p} b^{\alpha-(1 / p)}\left\|B u^{m}-B u^{0}\right\|_{L^{p}(J, Y)}
\end{aligned}
$$

Since $B$ is strongly continuous, we have

$$
\left\|B u^{m}-B u^{0}\right\|_{L^{p}(J, Y)} \stackrel{s}{\longrightarrow} 0 \quad \text { as } m \longrightarrow \infty,
$$

which implies

$$
\eta_{3}(t) \longrightarrow 0 \quad \text { as } m \longrightarrow \infty
$$

Thus

$$
\begin{aligned}
\left\|x^{m}(t)-x^{0}(t)\right\| \leq & \eta_{1}(t)+\eta_{3}(t) \\
& +M L_{f} \int_{0}^{t}\left\|x^{m}(s)-x^{0}(s)\right\| d s ;
\end{aligned}
$$

by virtue of singular version Gronwall inequality (i.e., Lemma 5), we obtain

$$
\left\|x^{m}(t)-x^{0}(t)\right\| \leq\left[\eta_{1}(t)+\eta_{3}(t)\right] E_{1}\left(M L_{f} b\right) .
$$

This yields that

$$
x^{m} \stackrel{s}{\longrightarrow} x^{0} \quad \text { in } P C(J, X) \text { as } m \longrightarrow \infty .
$$

Note that $H(6)$ implies all of the assumptions of Balder (see [18], Theorem 2.1) are satisfied. Hence, by Balders theorem, we can conclude that $(x, u) \rightarrow \int_{0}^{b} \mathscr{L}(t, x(t), u(t)) d t$ is sequentially lower semicontinuous in the strong topology of $L^{1}(J, X)$. Since $L^{p}(J, Y) \subset L^{1}(J, Y), \mathscr{J}$ is weakly lower semicontinuous on $L^{P}(J, Y)$, and since, by $H(6)($ iv), $\mathscr{J}>$ $-\infty, \mathcal{J}$ attains its infimum at $u_{0} \in U_{a d}$; that is,

$$
\begin{aligned}
\rho & =\lim _{m \rightarrow \infty} \int_{0}^{b} \mathscr{L}\left(t, x^{m}(t), u^{m}(t)\right) d t \\
& \geq \int_{0}^{b} \mathscr{L}\left(t, x^{0}(t), u^{0}(t)\right) d t=J\left(x^{0}, u^{0}\right) \geq \rho .
\end{aligned}
$$

The proof is completed.

\section{An Example}

Consider the following initial-boundary value problem of fractional impulsive parabolic control system

$$
\begin{gathered}
\frac{\partial^{\alpha}}{\partial t^{\alpha}} x(t, y) \\
=\frac{\partial^{2}}{\partial y^{2}} x(t, y)+I^{1-\alpha}\left(e^{-t}+\frac{1}{(t+10)} x(t, y)\right) \\
+\int_{0}^{1} q(y, \tau) u(\tau, t) d \tau, \quad t \in J^{\prime}=\frac{[0,1]}{\{1 / 2\}}, y \in[0, \pi], \\
\Delta x\left(\frac{1}{2}, y\right)=\frac{|x(y)|}{5+|x(y)|}, \quad y \in[0, \pi], \\
x(t, 0)=x(t, \pi)=0, \quad t \in J=[0,1], \\
x(0, y)=x_{0}(y), \quad y \in[0, \pi]
\end{gathered}
$$

with the cost function

$$
\mathscr{J}(x, u)=\int_{0}^{1} \int_{0}^{\pi}|x(t, y)|^{2} d y d t+\int_{0}^{1} \int_{0}^{\pi}|u(t, y)|^{2} d y d t
$$

where $\alpha=1 / 2, q:[0,1] \times[0,1] \rightarrow R$ is continuous, $u \in$ $L^{2}(J,[0,1]), b_{i} \in L^{2}(J)$.

Take $X=Y=L^{2}[0, \pi]$ and the operator $A: D(A) \subset$ $X \rightarrow X$ is defined by

$$
A \omega=\omega^{\prime \prime}
$$

where the domain $D(A)$ is given by

$$
\begin{aligned}
& \left\{\omega \in X: \omega, \omega^{\prime}\right. \text { are absolutely continuous, } \\
& \left.\omega^{\prime \prime} \in X, \omega(0)=\omega(\pi)=0\right\}
\end{aligned}
$$

Then $A$ can be written as

$$
A \omega=\sum_{n=1}^{\infty} n^{2}\left(\omega, \omega_{n}\right) \omega_{n}, \quad \omega \in D(A),
$$

where $\omega_{n}(x)=\sqrt{2 / \pi} \sin n x(n=1,2, \ldots)$ is an orthonormal basis of $X$. It is well known that $A$ is the infinitesimal 
generator of a compact semigroup $T(t)(t>0)$ in $X$ given by

$$
\begin{gathered}
T(t) x=\sum_{n=1}^{\infty} \exp ^{-n^{2} t}\left(x, x_{n}\right) x_{n}, \quad x \in X, \\
\|T(t)\| \leq e^{-1} \leq 1=M, \\
f(t, x(t, y))=e^{-t}+\frac{1}{(t+10)} x(t, y), \\
I_{k}(x(t, y))=\frac{|x(y)|}{5+|x(y)|}, \\
B(t, y)=\left[\int_{0}^{1} q(y, \tau) u(\tau, t) d \tau\right] .
\end{gathered}
$$

It is easy to see that

$$
\begin{gathered}
\|f(t, x(t))\| \leq \sqrt{\pi} e^{-t}+\frac{1}{10}\|x(t)\|, \\
\left\|I_{k}(x(t))\right\| \leq \frac{\|x(t)\|}{5}, \\
\|f(t, x(t))-f(t, y(t))\| \leq \frac{1}{10}\|x-y\|, \\
\left\|I_{1}(x(t))-I_{1}(y(t))\right\| \leq \frac{1}{5}\|x-y\|,
\end{gathered}
$$

and then

$$
\left(\sum_{i=1}^{1} h_{i}+\theta b\right) M=\left(\frac{1}{5}+\frac{1}{10} \times 1\right) \times 1<1 .
$$

Hence, all the conditions of Theorem 12 are satisfied, and system (58) has a unique optimal solution.

\section{Acknowledgments}

The authors thank the referees for their careful reading of the paper and insightful comments, which help to improve the quality of the paper. They would also like to acknowledge the valuable comments and suggestions from the editors, which vastly contributed to improve the presentation of the paper.

\section{References}

[1] K. S. Miller and B. Ross, An Introduction to the Fractional Calculus and Differential Equations, John Wiley \& Sons, New York, NY, USA, 1993.

[2] A. A. Kilbas, H. M. Srivastava, and J. J. Trujillo, Theory and Applications of Fractional Differential Equations, vol. 204 of North-Holland Mathematics Studies, Elsevier Science B.V., Amsterdam, The Netherlands, 2006.

[3] V. Lakshmikantham, S. Leela, and J. Vasundhara Devi, Theory of Fractional Dynamic Systems, Cambridge Academic Publishers, Cambridge, UK, 2009.

[4] K. Diethelm, The Analysis of Fractional Differential Equations, vol. 2004, Springer, Berlin, Germany, 2010.
[5] J. Wang, Y. Zhou, and M. Medved, "On the solvability and optimal controls of fractional integrodifferential evolution systems with infinite delay," Journal of Optimization Theory and Applications, vol. 152, no. 1, pp. 31-50, 2012.

[6] J. Wang, Y. Zhou, and W. Wei, "A class of fractional delay nonlinear integrodifferential controlled systems in Banach spaces," Communications in Nonlinear Science and Numerical Simulation, vol. 16, no. 10, pp. 4049-4059, 2011.

[7] X. B. Shu, Y. Lai, and Y. Chen, "The existence of mild solutions for impulsive fractional partial differential equations," Nonlinear Analysis, vol. 74, no. 5, pp. 2003-2011, 2011.

[8] M. Benchohra, J. Henderson, S. K. Ntouyas, and A. Ouahab, "Existence results for fractional order functional differential equations with infinite delay," Journal of Mathematical Analysis and Applications, vol. 338, no. 2, pp. 1340-1350, 2008.

[9] K. Balachandran, Y. Zhou, and J. Kokila, "Relative controllability of fractional dynamical systems with delays in control," Communications in Nonlinear Science and Numerical Simulation, vol. 17, no. 9, pp. 3508-3520, 2012.

[10] C. Cuevas and C. Lizama, "Almost automorphic solutions to a class of semilinear fractional differential equations," Applied Mathematics Letters, vol. 21, no. 12, pp. 1315-1319, 2008.

[11] E. Bazhlekova, Fractional evolution equations in Banach spaces [Ph.D. thesis], Eindhoven University of Technology, 2001.

[12] L. Xue and J. Xiong, "Existence and uniqueness of mild solutions for abstract delay fractional differential equations," Computers \& Mathematics with Applications, vol. 62, no. 3, pp. 1398-1404, 2011.

[13] H. Ye, J. Gao, and Y. Ding, "A generalized Gronwall inequality and its application to a fractional differential equation," Journal of Mathematical Analysis and Applications, vol. 328, no. 2, pp. 1075-1081, 2007.

[14] J. Wang, M. Fečkan, and Y. Zhou, "Relaxed controls for nonlinear fractional impulsive evolution equations," Journal of Optimization Theory and Applications, vol. 156, no. 1, pp. 13-32, 2013.

[15] S. Hu and N. S. Papageorgiou, Handbook of multivalued Analysis, Kluwer Academic Publishers, London, UK, 1997.

[16] J. Wang, M. Fečkan, and Y. Zhou, "On the new concept of solutions and existence results for impulsive fractional evolution equations," Dynamics of Partial Differential Equations, vol. 8, no. 4, pp. 345-361, 2011.

[17] Y. Zhou and F. Jiao, "Existence of mild solutions for fractional neutral evolution equations," Computers \& Mathematics with Applications, vol. 59, no. 3, pp. 1063-1077, 2010.

[18] E. J. Balder, "Necessary and sufficient conditions for $L_{1}$-strongweak lower semicontinuity of integral functionals," Nonlinear Analysis, vol. 11, no. 12, pp. 1399-1404, 1987. 


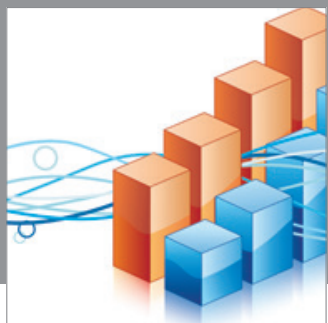

Advances in

Operations Research

mansans

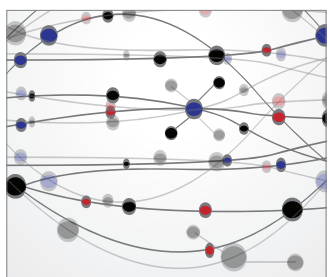

The Scientific World Journal
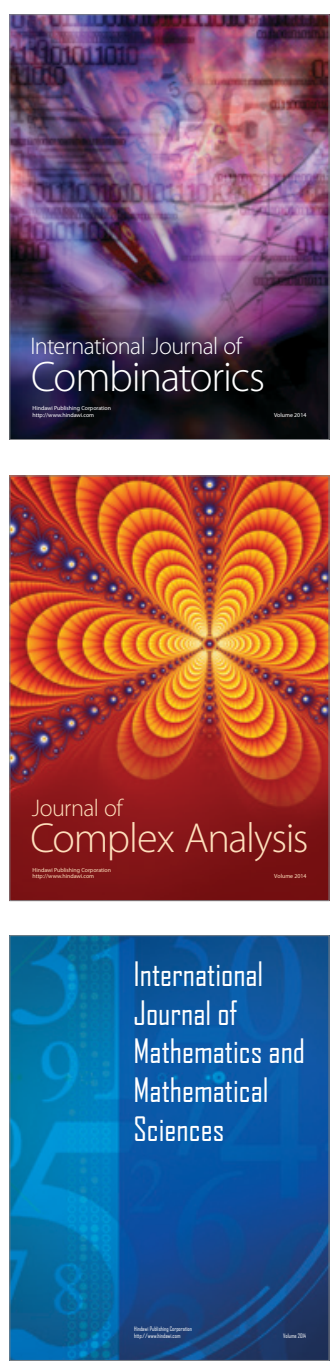
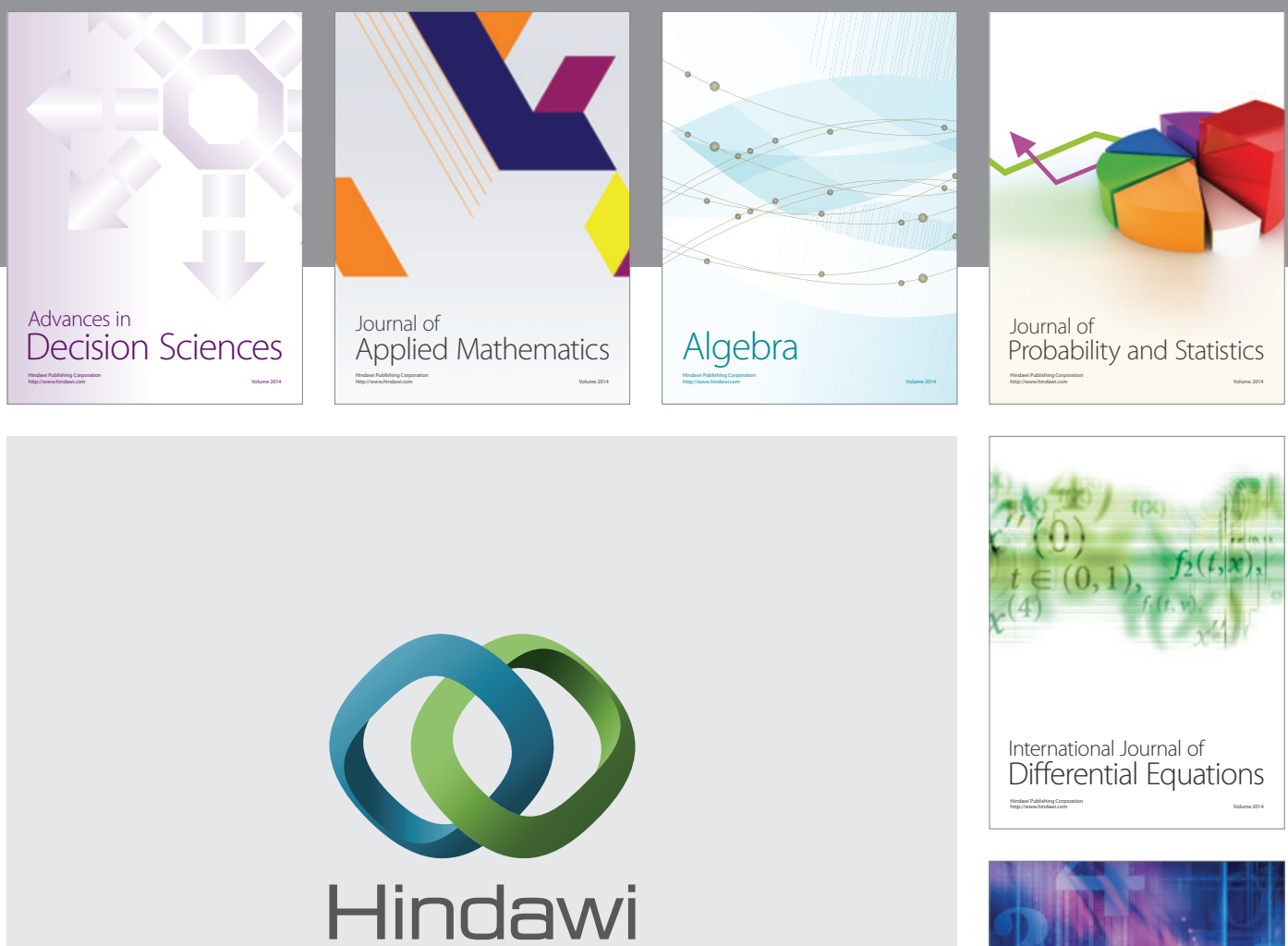

Submit your manuscripts at http://www.hindawi.com
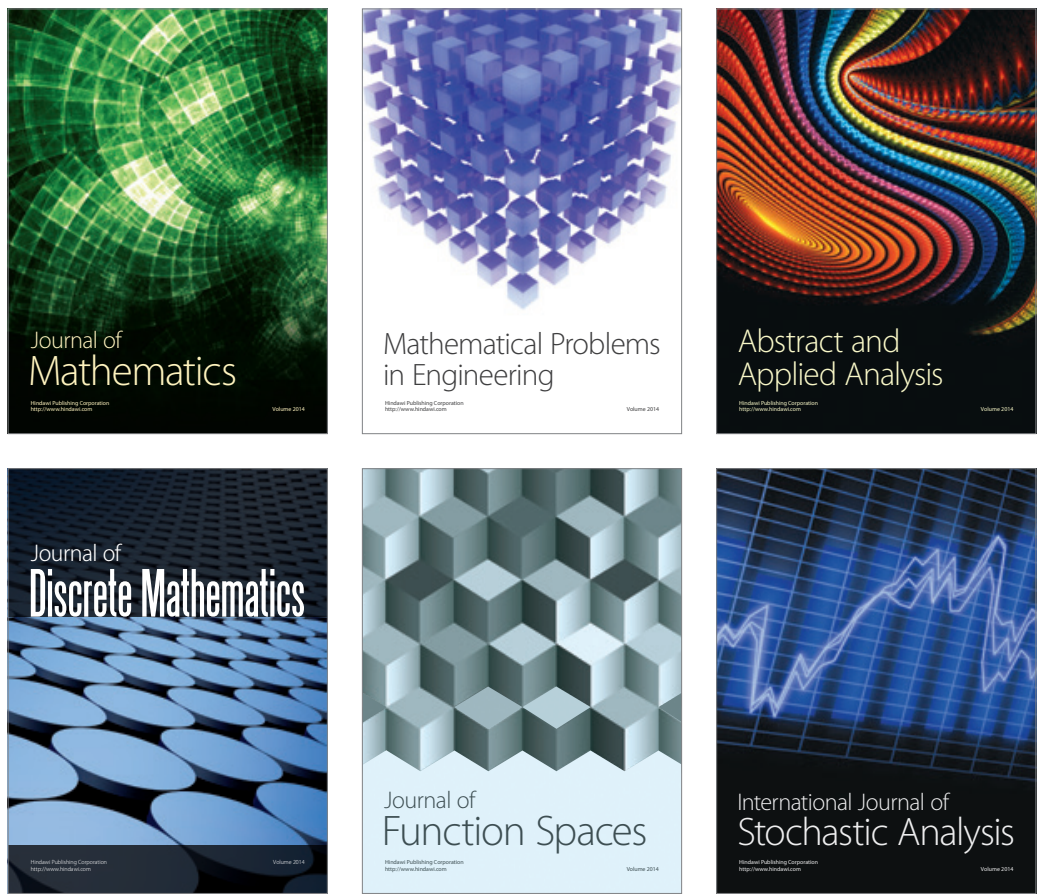

Journal of

Function Spaces

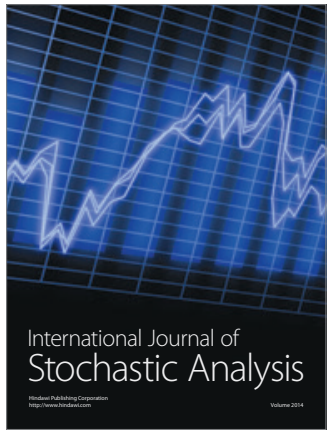

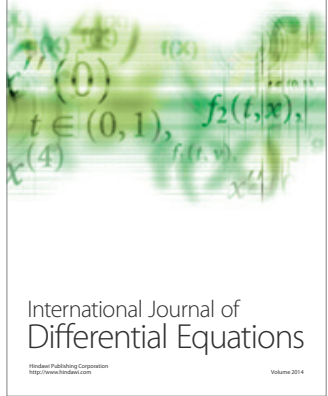
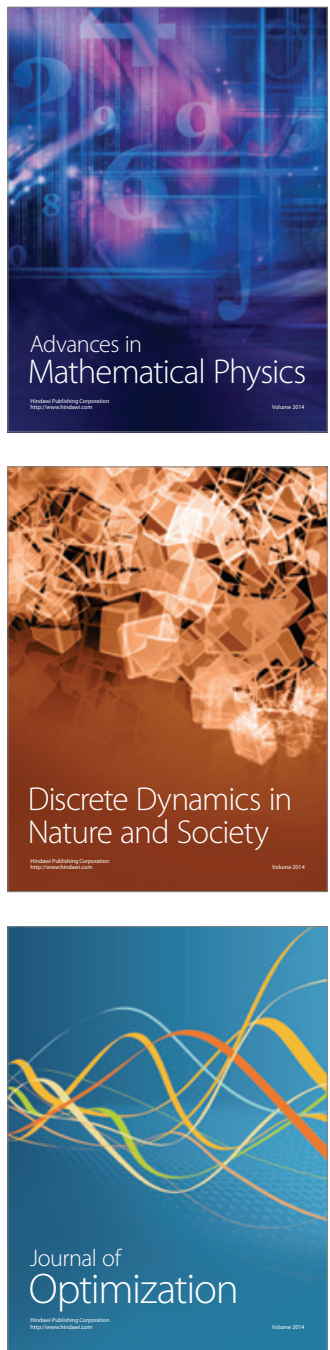